\title{
Solving a Two-stage Continuous-discrete Problem of Optimal Partitioning-Allocation with Subsets Centers Placement
}

https://doi.org/10.1515/comp-2020-0142

Received Sep 04, 2019; accepted Apr 06, 2020

Abstract: A two-stage continuous-discrete optimal partitioning-allocation problem is studied, and a method and an algorithm for its solving are proposed. This problem is a generalization of a classical transportation problem to the case when coordinates of the production points (collection, storage, processing) of homogeneous products are continuously allocated in the given domain and the production volumes at these points are unknown. These coordinates are found as a solution of the corresponding continuous optimal set-partitioning problem in a finitedimensional Euclidean space with the placement (finding coordinates) of these subsets' centers. Also, this problem generalizes discrete two-stage production-transportation problems to the case of continuously allocated consumers. The method and algorithm are illustrated by solving two model problems.

Keywords: infinite-dimensional mathematical programming, the theory of optimal set-partitioning, transportation problem, non-differentiable optimization, Shor's $r$ algorithm.

2010 Mathematics Subject Classification: 49M29, 49J52, $49 \mathrm{~K} 35$

\footnotetext{
*Corresponding Author: Liudmyla Hart: Department of Computational Mathematics and Mathematical Cybernetics, Oles Honchar Dnipro National University (DNU), Dnipro, 49010, Ukraine; Email: ll_hart@ukr.net

Elena Kiseleva: Faculty of Applied Mathematics, Oles Honchar Dnipro National University (DNU), Dnipro, 49010, Ukraine; Email: kiseleva47@ukr.net

Olha Prytomanova: Department of Computational Mathematics and Mathematical Cybernetics, Oles Honchar Dnipro National University (DNU), Dnipro, 49010, Ukraine; Email: olgmp@ua.fm
}

\section{Introduction}

The mathematical theory of optimal set-partitioning (OSP) in $n$-dimensional Euclidean space is a new non-classical area of infinite-dimensional mathematical programming operating with Boolean variables.

Studies on the optimal set-partitioning topic were started in the 1970s by scientists H. Corley and S. Roberts $[1,2]$ and, independently, by mathematicians I. Beyko and E. Kiseleva [3, 4]. In their studies, H. Corley and S. Roberts were only able to get the necessary conditions for optimality of partitions. At the same time, I. Beyko and E. Kiseleva managed to advance further and to develop numerical algorithms for finding optimal solutions based on similar necessary optimality conditions obtained by them. This was made possible by using effective methods of non-differentiable optimization (various variants of the Shor's $r$-algorithm [5-8]) for the numerical solution of auxiliary finite-dimensional optimization problems that arise during the development of methods for solving infinitedimensional OSP problems.

Numerous important theoretical and practical optimization problems, that are completely different, are reducible to continuous models of optimal set-partitioning. Here, let us indicate a few of them: the generalized Neumann-Pearson problems [9]; various global optimization problems [10]; a problem of forming optimal quadratures [9]; a problem of constructing the Dirichlet-Voronoi diagram and its generalizations $[9,11,12]$; optimal ball covering continuous problems [13] and other optimal geometric objects' packing $[14,15]$, etc.

Also, the OSP theory can be used for solving applied problems such as pattern recognition with a goal to minimize the average function of wrong recognition; medical diagnostics aiming to minimize errors of diagnosis; service sector territorial planning; geological forecasting; environment protection, e.g., a problem of providing environmental safety of placing radioactive waste disposal facilities of nuclear power plants, taking into account an ecological structure of the placement domain; placement of am- 
bulance stations, cellular base stations, oil wells, etc. A typical optimal set-partitioning continuous problem is an infinite-dimensional transportation problem or its generalizations, such as infinite-dimensional problems of enterprises' placement with simultaneous partitioning of a certain domain, continuously allocated by consumers, into sub-domains served by one enterprise, in order to minimize transportation and production costs, the so-called location-allocation problems [16]. Here, consumers can be telephone subscribers, schoolchildren, voters, points of irrigated territory, patients, etc.

Compared with the work [16] and the works indicated in the references in [16], the algorithms developed in the OSP theory have a number of advantages:

- they are applicable for solving non-linear, multiproduct location-allocation problems with restrictions on the capacity of enterprises in the form of equalities and inequalities with the enterprises' placement;

- they allow us to obtain optimal partitions of the set of consumers into sub-domains of their service by each of the enterprises on each product; optimal coordinates of the placed enterprises and optimal capacities of enterprises;

- they do not depend on the dimension of space $E_{n}$ containing the partitionable domain (the problem is only reduced to the calculation of multidimensional integrals);

- they do not depend on the geometry of the partitionable domain;

- due to the high calculation speed of the Shor's $r$ algorithm, they are applicable for solving problems of large dimensions $(100,200,300$ or more subdomains) [9];

- during the iterative process, the location of subdomains' centers, partitions and production volumes at enterprises are simultaneously improved.

A comprehensive list of optimization problems, reducible to OSP problems, the corresponding references, and a description of the modern optimal set-partitioning theory are given in paper [9]. Certain areas of this theory are:

- deterministic linear and nonlinear, single-product and multi-product OSP problems under constraints, both with a given placement of the subsets' centers and with finding the best option for their location;

- problems of optimal set-partitioning under conditions of uncertainty. To remove the uncertainty, one can use either the mathematical apparatus of stochastic infinite-dimensional mathematical programming (if part of the initial information is probabilistic) or the apparatus of fuzzy sets and fuzzy logic (if the parameters of the models are fuzzy, inaccurate, unreliable, etc.);

- dynamic problems of optimal set-partitioning with an optimality criterion depending on phase trajectories and on the control of some given controlled system;

- continuous problems on spherical coating, reducible to the OSP problems.

This paper is dedicated to the further development of the optimal set-partitioning theory in $n$-dimensional Euclidean space into subsets for the case of two-stage continuous-discrete optimal partitioning-allocation problem under constraints in the form of equalities and with placement (finding of coordinates) of subset centers in a given (partitionable) set.

On the one hand, this continuous-discrete optimal partitioning-allocation problem is a generalization of the classical transportation problem [17] for the case when coordinates of the points of production (collection, storage, processing) of homogeneous products are continuously allocated in a given set. Production volumes at these points are apriori unknown and are searched as a solution to the corresponding continuous problem of optimal setpartitioning into subsets with a placement (finding of optimal coordinates) of the subsets' centers. A simpler twostage continuous-discrete optimal partitioning-allocation problem with given positions of the subsets' centers was presented in [18]. On the other hand, the continuousdiscrete partitioning-allocation problem generalizes discrete two-stage production-transportation problems [1923] for the case of continuously allocated consumers.

Real-world problems, reducible to two-stage continuous-discrete problems of optimal partitioningallocation with the placement of the subsets' centers, contain two stages. In the first stage, the goal is to determine the regions of collection of a continuously allocated resource (raw material) by enterprises. On the second stage, the goal is to determine enterprises' locations and transportation volumes of the products sent to consumers in order to minimize the total cost of transportation of the resources transferred from suppliers to processing points (collection, storage) and then to consumers. Note that such problems can be easily found in real-life [18]: these are ones where, for example, natural raw materials (oil, gas, ore) or the harvesting crops can be represented as a continuously allocated resource; the problem of organizing wood waste collection for fuel production with 
its subsequent distribution between points of heat energy production while minimizing the total transportation cost; problems of optimizing the deposit and credit activity of bank branches aiming to attract deposits from individuals with a subsequent allocation of the funds between borrowers, and many others.

A solution of two-stage continuous-discrete optimal partitioning-allocation problem can be found based on the general approach [9]. The approach lies in reducing the original infinite-dimensional problems of optimal partitioning-allocation to non-smooth, typically finitedimensional, optimization problems. For numerical solving these non-smooth optimization problems, effective methods of non-differentiable optimization can be used, such as different variants of the Shor's $r$-algorithm [5-8].

For the problem under consideration, a solution method is proposed, which is based on the use of OSP methods and the potential method for solving the transportation problem. Based on the proposed method, a solution algorithm was developed, which uses a modification of the $r$-algorithm [13-16]. Results of computational experiments that were conducted using the developed algorithm are given for two model problems

\section{Methods}

In this section, we present the statement of a two-stage continuous-discrete linear single-product problem of optimal partitioning-allocation with the placement (finding coordinates) of subsets' centers subject to equality constraints. We also provide a justification for a method to solve such a problem and formulate a numerical algorithm for its solution.

\subsection{Problem statement}

Let $\Omega$ be a bounded, closed, Lebesgue measurable set in $n$-dimensional Euclidean space $E_{n}$.

We will refer to a set of Lebesgue measurable subsets $\Omega_{1}, \ldots, \Omega_{N}$ of $\Omega \subset E_{n}$ as a possible partitioning set $\Omega$ into its non-intersecting subsets, if

$$
\bigcup_{i=1}^{N} \Omega_{i}=\Omega, \text { mes }\left(\Omega_{i} \cap \Omega_{j}\right)=0, i, j=1,2, \ldots, N(i \neq j),
$$

where $m e s(\cdot)$ is a Lebesgue measure.
By $\sum_{\Omega}^{N}$, we denote the class of all possible partitions of a set $\Omega$ into subsets $\Omega_{1}, \ldots, \Omega_{N}$, that is

$$
\begin{aligned}
& \sum_{\Omega}^{N}=\left\{\left\{\Omega_{1}, \ldots, \Omega_{N}\right\}: \bigcup_{i=1}^{N} \Omega_{i}=\Omega, \text { mes }\left(\Omega_{i} \cap \Omega_{j}\right)=0,\right. \\
& i, j=1,2, \ldots, N(i \neq j)\} .
\end{aligned}
$$

We introduce a functional

$$
\begin{aligned}
& F\left(\left\{\Omega_{1}, \ldots, \Omega_{N}\right\},\left\{\tau_{1}^{I}, \ldots, \tau_{N}^{I}\right\},\left\{v_{11}, \ldots, v_{N M}\right\}\right) \\
& =\sum_{i=1}^{N} \int_{\Omega_{i}} c_{i}^{I}\left(x, \tau_{i}^{I}\right) \rho(x) d x+\sum_{i=1}^{N} \sum_{j=1}^{M} c_{i j}^{I I}\left(\tau_{i}^{I}, \tau_{j}^{I I}\right) v_{i j},
\end{aligned}
$$

where $\tau_{1}^{I}, \ldots, \tau_{N}^{I}$ is a set of some reference points for subsets $\Omega_{1}, \ldots, \Omega_{N}$ called centers of these subsets $\left(\tau_{i}^{I} \in \Omega_{i}\right.$, $i=1,2, \ldots, N)$, which coordinates $\tau_{i}^{I}=\left(\tau_{i}^{I(1)}, \ldots, \tau_{i}^{I(n)}\right)$, $i=1,2, \ldots, N$, are unknown and require their finding; $\tau_{1}^{I I}, \ldots, \tau_{M}^{I I}$ are certain points of set $\Omega ; v_{i j}(i=1,2, \ldots, N$, $j=1,2, \ldots, M)$ are weight parameters specifying a tightness of the connection between the centers of subsets $\tau_{i}^{I} \in \Omega_{i}, i=1,2, \ldots, N$ and the points $\tau_{j}^{I I} \in \Omega, \tau_{j}^{I I}=$ $\left(\tau_{j}^{I I(1)}, \ldots, \tau_{j}^{I I(n)}\right), j=1,2, \ldots, M . \rho(x) \geq 0$ is a given function, bounded and measurable on $\Omega ; c_{i}^{I}\left(x, \tau_{i}^{I}\right), i=$ $1,2, \ldots, N$, are given real functions defined and bounded on $\Omega \times \Omega$, measurable by argument $x=\left(x^{(1)}, \ldots, x^{(n)}\right)$ for any fixed $\tau_{i}^{I}=\left(\tau_{i}^{I(1)}, \ldots, \tau_{i}^{I(n)}\right) \in \Omega_{i} \subset \Omega ; c_{i j}^{I I}\left(\tau_{i}^{I}, \tau_{j}^{I I}\right)$, $j=1,2, \ldots, M$, are given functions defined and bounded on $\Omega \times \Omega$, representing "distance" between points $\tau_{i}^{I} \in \Omega_{i}$ and $\tau_{j}^{I I} \in \Omega$ at the corresponding metric of space $E_{n}$.

To the following problem, we will refer to as a twostage continuous-discrete linear single-product problem of optimal partitioning-allocation with the placement (finding coordinates) of subsets' centers subject to equality constraints.

Problem A. It is required to find a partition of a set $\Omega$ into $N$ Lebesgue-measurable subsets $\Omega_{\star_{1}}, \ldots, \Omega_{\star_{i}}, \ldots, \Omega_{\star_{N}}$, coordinates of centers $\tau_{\star_{1}}^{I}, \ldots, \tau_{\star_{i}}^{I}, \ldots, \tau_{\star_{N}}^{I}$ of these subsets in the domain $\Omega$, and a non-negative vector $v_{\star}=$ $\left(v_{\star_{11}}, \ldots, v_{\star_{i j}}, \ldots, v_{\star_{N M}}\right) \in E_{N M}$, which provide

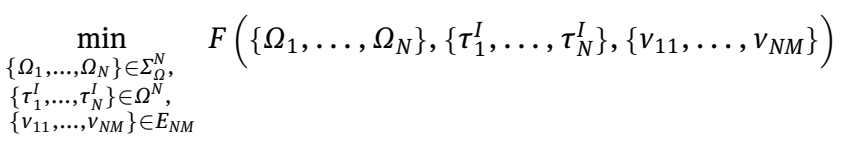

subject to constraints

$$
\sum_{j=1}^{M} v_{i j}=\int_{\Omega_{i}} \rho(x) d x, i=1, \ldots, N ; \sum_{i=1}^{N} v_{i j}=b_{j}^{I I},
$$


$j=1, \ldots, M ;\left\{\Omega_{1}, \ldots, \Omega_{N}\right\} \in \Sigma_{\Omega}^{N} ; v_{i j} \geq 0, i=1, \ldots, N$,

$j=1, \ldots, M ; x=\left(x^{(1)}, \ldots, x^{(n)}\right) \in \Omega ; \tau^{I}=\left(\tau_{1}^{I}, \ldots, \tau_{N}^{I}\right)$

$$
\in \underbrace{\Omega \times \ldots \times \Omega}_{N}=\Omega^{N}, \tau^{I I}=\left(\tau_{1}^{I I}, \ldots, \tau_{M}^{I I}\right) \in \Omega^{M} .
$$

Here $b_{j}^{I I}, j=1, \ldots, M$, are given non-negative numbers, herewith the solvability conditions of the problem hold:

$$
\begin{aligned}
& S=\int_{\Omega} \rho(x) d x=\sum_{i=1}^{N} \int_{\Omega_{i}} \rho(x) d x=\sum_{i=1}^{N} \sum_{j=1}^{M} v_{i j}=\sum_{j=1}^{M} b_{j}^{I I}, \\
& 0 \leq b_{j}^{I I} \leq S, j=1, \ldots, M .
\end{aligned}
$$

Note that, in terms of the classical transportation problem, a vector $v=\left(v_{11}, \ldots, v_{N M}\right)$ has the meaning of a volume of products transportation from points $\tau_{i}^{I}, i=1, \ldots, N$, of the first stage, to points $\tau_{j}^{I I}, j=1, \ldots, M$, of final consumption (the second stage).

Here and in the following, we consider Lebesgue integrals. We assume that the measure of the set of boundary points of subsets $\Omega_{i}, i=1, \ldots, N$, is equal to zero.

We define a triple $\left(\left\{\Omega_{\star_{1}}, \ldots, \Omega_{\star_{N}}\right\},\left\{\tau_{\star_{1}}^{I}, \ldots, \tau_{\star_{N}}^{I}\right\}\right.$ $\left.\left\{v_{\star} 11, \ldots, v_{\star} N M\right\}\right)$, which minimizes functional $F$ on a set $\Sigma_{\Omega}^{N} \times \Omega^{N} \times E_{N M}$, as an optimal solution to Problem A. Wherein, in Problem A. we define a partition $\left\{\Omega_{\star_{1}}, \ldots, \Omega_{\star_{N}}\right\} \in \Sigma_{\Omega}^{N}$ as an optimal partitioning of set $\Omega \subset E_{n}$ into $N$ subsets, and a set $\tau_{\star}^{I}=\left\{\tau_{\star_{1}}^{I}, \ldots, \tau_{\star_{N}}^{I}\right\} \in \Omega^{N}$ of centers $\tau_{\star_{i}}^{I} \in \Omega_{\star_{i}}, i=1,2, \ldots, N$ as optimal centers of subsets $\Omega_{\star i}, i=1,2, \ldots, N$.

We introduce characteristic functions

$$
\lambda_{i}(x)=\left\{\begin{array}{l}
1, x \in \Omega_{i}, \\
0, x \in \Omega \backslash \Omega_{i},
\end{array} \quad i=1, \ldots, N,\right.
$$

of subsets $\Omega_{i}, i=1, \ldots, N$, respectively, and consider a functional

$$
\begin{aligned}
I\left(\lambda(\cdot), \tau^{I}, v\right) & =\sum_{i=1}^{N} \int_{\Omega} c_{i}^{I}\left(x, \tau_{i}^{I}\right) \rho(x) \lambda_{i}(x) d x \\
& +\sum_{i=1}^{N} \sum_{j=1}^{M} c_{i j}^{I I}\left(\tau_{i}^{I}, \tau_{j}^{I I}\right) v_{i j}
\end{aligned}
$$

where $\lambda(\cdot)=\left(\lambda_{1}(\cdot), \ldots, \lambda_{i}(\cdot), \ldots, \lambda_{N}(\cdot)\right)$ is a vector function defined on $\Omega ; \tau^{I}=\left(\tau_{1}^{I}, \ldots, \tau_{i}^{I}, \ldots, \tau_{N}^{I}\right) \in \Omega^{N}, v=$ $\left(v_{11}, \ldots, v_{i j}, \ldots, v_{N M}\right) \in E_{N M}$. Obviously,

$$
\begin{aligned}
& I\left(\lambda(\cdot), \tau^{I}, v\right)=F\left(\left\{\Omega_{1}, \ldots, \Omega_{N}\right\},\left\{\tau_{1}^{I}, \ldots, \tau_{N}^{I}\right\},\right. \\
& \left.\left\{v_{11}, \ldots, v_{N M}\right\}\right) .
\end{aligned}
$$

Let rewrite Problem A in terms of characteristic functions $\lambda_{i}(x)$ of subsets $\Omega_{i}, i=1, \ldots, N$, in the following form.
Problem B. It is required to find $\min _{\left(\lambda(\cdot), \tau^{I}, v\right)} I\left(\lambda(\cdot), \tau^{I}, v\right)$ under constraints

$$
\begin{aligned}
& \sum_{j=1}^{M} v_{i j}=\int_{\Omega} \rho(x) \lambda_{i}(x) d x, i=1, \ldots, N ; \sum_{i=1}^{N} v_{i j}=b_{j}^{I I}, \\
& j=1, \ldots, M ;
\end{aligned}
$$

$\lambda_{i}(x)=0 \vee 1$ almost everywhere (a.e.) for $x \in \Omega, i=$ $1, \ldots, N ; \sum_{i=1}^{N} \lambda_{i}(x)=1$ a.e. for $x \in \Omega$;

$$
\begin{aligned}
& \tau^{I}=\left(\tau_{1}^{I}, \ldots, \tau_{i}^{I}, \ldots, \tau_{N}^{I}\right) \in \Omega^{N} ; v_{i j} \geq 0, i=1, \ldots, N, \\
& j=1, \ldots, M .
\end{aligned}
$$

From the infinite-dimensional Problem $B$ with Boolean values of variables $\lambda_{i}(\cdot), i=1, \ldots, N$, let us proceed to the corresponding problem with function $\lambda_{i}(\cdot)$ values from the segment $[0,1]$.

Problem C. It is required to find

$$
\min _{\left(\lambda(\cdot), \tau^{I}, v\right) \in \Gamma_{1} \times \Omega^{N} \times Q} I\left(\lambda(\cdot), \tau^{I}, v\right),
$$

where

$$
\begin{aligned}
& \Gamma_{1}=\left\{\lambda(x)=\left(\lambda_{1}(x), \ldots, \lambda_{N}(x)\right) \in \Gamma \text { a.e. for } x \in \Omega:\right. \\
& \sum_{j=1}^{M} v_{i j}=\int_{\Omega} \rho(x) \lambda_{i}(x) d x, i=1, \ldots, N ; \\
& \left.\sum_{i=1}^{N} v_{i j}=b_{j}^{I I}, j=1, \ldots, M\right\} ;
\end{aligned}
$$

$$
\begin{aligned}
& \Gamma=\left\{\lambda(x): 0 \leq \lambda_{i}(x) \leq 1 \forall x \in \Omega, i=1, \ldots, N ;\right. \\
& \left.\sum_{i=1}^{N} \lambda_{i}(x)=1 \text { a.e. for } x \in \Omega\right\} ; \\
& \tau^{I}=\left(\tau_{1}^{I}, \ldots, \tau_{i}^{I}, \ldots, \tau_{N}^{I}\right) \in \Omega^{N} ; \\
& Q=\left\{v=\left(v_{11}, \ldots, v_{i j}, \ldots, v_{N M}\right):\right. \\
& \left.v_{i j} \geq 0, i=1, \ldots, N, j=1, \ldots, M\right\} .
\end{aligned}
$$

For each fixed $v \in Q$, Problem $C$ has a solution as it is proved in [9].

Indeed, since $\Gamma_{1}$ is a bounded, closed, convex set of Hilbert space $L_{2}^{N}(\Omega)$, and the functional $I\left(\lambda(\cdot), \tau^{I}, v\right)$ is linear 
(and therefore convex) and continuous by $\lambda(\cdot)$ on $\Gamma_{1}$ for each fixed point $\tau^{I} \in \Omega^{N}$ and each fixed point $v \in Q$, then, by the known generalized theorem of Weierstrass, a convex continuous functional $I\left(\lambda(\cdot), \tau^{I}, v\right)$ under fixed $\tau^{I} \in \Omega^{N}$ and fixed $v \in Q$ reaches its lower bound by $\lambda(\cdot)$ on a closed bounded convex set $\Gamma_{1}$ of Hilbert space $L_{2}^{N}(\Omega)$.

Statement 2.1. For every fixed $\tau^{I} \in \Omega^{N}$ and every fixed $v \in$ $Q$, the bounded, closed, convex set $\Gamma_{1}$ of the Hilbert space $L_{2}^{N}(\Omega)$ is weakly compact and (according to the known Kerin - Milman theorem) contains at least one extreme point.

Statement 2.2. Among points of set $\Gamma_{1}$, where a linear by $\lambda(\cdot)$ functional $I\left(\lambda(\cdot), \tau^{I}, v\right)$ attains its minimal value by $\lambda(\cdot)$ on set $\Gamma_{1}$, for each fixed $\tau^{I} \in \Omega^{N}$ and each fixed $v \in Q$, there exists at least one extreme point of set $\Gamma_{1}$.

Statement 2.3. Extreme points of a set $\Gamma_{1}$ are characteristic functions of some subsets $\Omega_{i}, i=1, \ldots, N$, that form a partition of set $\Omega$ for each fixed $\tau^{I} \in \Omega^{N}$ and each fixed $v \in Q$.

Statements $2.1-2.3$ imply that, for each fixed $\tau^{I} \in \Omega^{N}$ and each fixed $v \in Q$, in the set of optimal solutions of Problem $C$, there exist optimal solutions to Problem B, which allows us to proceed to the consideration of Problem C.

\subsection{Justification of a Problem Solution Method}

Let introduce a Lagrangian for Problem C as follows:

$$
\begin{aligned}
& L\left(\left\{\lambda(\cdot), \tau^{I}, v\right\}, \Psi\right)=I\left(\lambda(\cdot), \tau^{I}, v\right) \\
& +\sum_{i=1}^{N} \psi_{i}\left(\int_{\Omega} \rho(x) \lambda_{i}(x) d x-\sum_{j=1}^{M} v_{i j}\right) \\
& +\sum_{j=1}^{M} \eta_{j}\left(b_{j}^{I I}-\sum_{i=1}^{N} v_{i j}\right)
\end{aligned}
$$

where $\Psi=\left(\psi_{1}, \ldots, \psi_{i}, \ldots, \psi_{N} ; \eta_{1}, \ldots, \eta_{j}, \ldots, \eta_{M}\right)$ is an $(N+M)$-dimensional real vector with coordinates of an arbitrary sign; $\lambda(x)=\left(\lambda_{1}(x), \ldots, \lambda_{N}(x)\right) \in \Gamma$ a.e. for $x \in \Omega ; \tau^{I}=\left(\tau_{1}^{I}, \ldots, \tau_{i}^{I}, \ldots, \tau_{N}^{I}\right) \in \Omega^{N} ; v=$ $\left(v_{11}, \ldots, v_{i j}, \ldots, v_{N M}\right)$ is a $N \times M$-dimensional real nonnegative vector.

To a pair of elements $\left(\left\{\lambda_{\star}(\cdot), \tau_{\star}^{I}, v_{\star}\right\}, \Psi^{\star}\right)$, we will refer to as a saddle point of functional (2) on set $\left\{\Gamma \times \Omega^{N} \times Q\right\} \times \Lambda$, where

$$
\Lambda=\left\{\Psi=(\psi ; \eta) \in E_{N+M}: \psi=\left(\psi_{1}, \ldots, \psi_{N}\right) \in E_{N},\right.
$$

$$
\left.\eta=\left(\eta_{1}, \ldots, \eta_{M}\right) \in E_{M}\right\},
$$

if $L\left(\left\{\lambda_{\star}(\cdot), \tau_{\star}^{I}, v_{\star}\right\}, \Psi\right) \leq L\left(\left\{\lambda_{\star}(\cdot), \tau_{\star}^{I}, v_{\star}\right\}, \Psi^{\star}\right) \leq$ $L\left(\left\{\lambda(\cdot), \tau^{I}, v\right\}, \Psi^{\star}\right)$ for $\lambda(\cdot) \in \Gamma, \tau^{I} \in \Omega^{N}, v \in Q, \Psi \in \Lambda$, i.e.,

$$
\begin{aligned}
& L\left(\left\{\lambda_{\star}(\cdot), \tau_{\star}^{I}, v_{\star}\right\}, \Psi^{\star}\right)=\min _{\left\{\lambda(\cdot), \tau^{I}, v\right\} \in \Gamma \times \Omega^{N} \times Q} \max _{\Psi \in \Lambda} L(\{\lambda(\cdot), \\
& \left.\left.\tau^{I}, v\right\}, \Psi\right)=\max _{\Psi \in \Lambda} \min _{\left\{\lambda(\cdot), \tau^{I}, v\right\} \in \Gamma \times \Omega^{N} \times Q} L\left(\left\{\lambda(\cdot), \tau^{I}, v\right\}, \Psi\right) .
\end{aligned}
$$

Let us introduce functionals

$$
\begin{gathered}
X\left(\left\{\lambda(\cdot), \tau^{I}, v\right\}\right)=\max _{\Psi \in \Lambda} L\left(\left\{\lambda(\cdot), \tau^{I}, v\right\}, \Psi\right), \\
\left\{\lambda(\cdot), \tau^{I}, v\right\} \in \Gamma \times \Omega^{N} \times Q ; \\
G(\Psi)=\min _{\left\{\lambda(\cdot), \tau^{I}, v\right\} \in \Gamma \times \Omega^{N} \times Q} L\left(\left\{\lambda(\cdot), \tau^{I}, v\right\}, \Psi\right), \Psi \in \Lambda
\end{gathered}
$$

and consider problems

$$
\begin{aligned}
& X\left(\left\{\lambda(\cdot), \tau^{I}, v\right\}\right) \rightarrow \min ,\left\{\lambda(\cdot), \tau^{I}, v\right\} \in \Gamma \times Q^{N} \\
& \times Q . \\
& G(\Psi) \rightarrow \max , \Psi \in \Lambda .
\end{aligned}
$$

Let us call the problem (3) as the primal one, and the problem (4) as dual to the problem (3).

By analogy with [9], it is easy to show that problems (3), (4) satisfy the duality relation $X_{\star}=G^{\star}$ and solving a pair of dual problems (3) and (4) (both are solvable) is equivalent to finding a saddle point of the Lagrangian (2) on set $\left\{\Gamma \times \Omega^{N} \times Q\right\} \times \Lambda$.

To find a saddle point of the Lagrangian (2), let us specify the dual problem (4). For this, according to [9], we move from the problem of finding $\min _{\left.\tau^{I}\right) \in \Gamma \times \Omega^{N}, v \in Q} L\left(\left\{\lambda(\cdot), \tau^{I}, v\right\}, \Psi\right)$ to the following problem: for each fixed $\Psi \in \Lambda$, it is required to find:

$$
\min _{\tau^{I} \in \Omega^{N}} \min _{v \geq 0} \min _{\lambda(\cdot) \in \Gamma} L\left(\left\{\lambda(\cdot), \tau^{I}, v\right\}, \Psi\right) .
$$

Denote

$$
\begin{aligned}
& G_{1}\left(\tau^{I}, v, \Psi\right)=G_{1}\left(\tau^{I}, v,\{\psi, \eta\}\right) \\
& =\min _{\lambda(\cdot) \in \Gamma} L\left(\left\{\lambda(\cdot), \tau^{I}, v\right\}, \Psi\right)
\end{aligned}
$$

Substituting in (5) $L\left(\left\{\lambda(\cdot), \tau^{I}, v\right\}, \Psi\right)$ by the formula from (2), as well as taking into account (1) and the fact that functional (2) is linearly separable by $\lambda$ (for each fixed $\tau^{I} \in \Omega^{N}$ and each fixed $v \geq 0$ ) on set $\Gamma$ [9], we obtain:

$$
G_{1}\left(\tau^{I}, v,\{\psi, \eta\}\right)=\sum_{j=1}^{M} \eta_{j} b_{j}^{I I}
$$




$$
\begin{aligned}
& +\int_{\Omega} \sum_{i=1}^{N} \min _{\substack{\sum_{i=1}^{N} \lambda_{i}=1, 0 \leq \lambda_{i} \leq 1, i=1, \ldots, N}}\left\{\left(c_{i}^{I}\left(x, \tau_{i}^{I}\right)+\psi_{i}\right) \rho(x) \lambda_{i}(x)\right\} d x \\
& +\sum_{i=1}^{N} \sum_{j=1}^{M}\left(c_{i j}^{I I}\left(\tau_{i}^{I}, \tau_{j}^{I I}\right)-\psi_{i}-\eta_{j}\right) v_{i j}
\end{aligned}
$$

It is easy to observe that, in (6), for each $\tau^{I} \in \Omega^{N}, v \geq 0$ and each $\Psi=(\psi, \eta) \in \Lambda$, the minimum value of the $i$-th expression $(i=1, \ldots, N)$ in curly brackets is attained at $\lambda_{i}(x)=\lambda_{\star i}(x)[9]$, where

$$
\lambda_{\star_{i}}(x)= \begin{cases}1, & c_{i}^{I}\left(x, \tau_{i}^{I}\right)+\psi_{i} \leq c_{k}^{I}\left(x, \tau_{k}^{I}\right)+\psi_{k}, \\ & i \neq k \text { a.e. for } x \in \Omega, k=1, \ldots, N, \\ 0, & \text { otherwise. }\end{cases}
$$

Then, taking into account the notation

$$
G_{2}\left(\tau^{I},\{\psi, \eta\}\right)=\min _{v \geq 0} G_{1}\left(\tau^{I}, v,\{\psi, \eta\}\right),
$$

the functional $G(\Psi)$ in the problem (4) takes the form of

$$
\begin{aligned}
& G(\Psi)=G(\psi, \eta)=\min _{\tau^{I} \in \Omega^{N}} G_{2}\left(\tau^{I},\{\psi, \eta\}\right) \\
& =\min _{\tau^{I} \in \Omega^{N}}\left[\int_{\Omega} \min _{k=1, N}\left(c_{k}^{I}\left(x, \tau_{k}^{I}\right)+\psi_{k}\right) \rho(x) d x\right. \\
& \left.+\sum_{j=1}^{M} \eta_{j} b_{j}^{I I}+\sum_{i=1}^{N} \sum_{j=1}^{M} \min _{v_{i j} \geq 0}\left(c_{i j}^{I I}\left(\tau_{i}^{I}, \tau_{j}^{I I}\right)-\psi_{i}-\eta_{j}\right) v_{i j}\right]
\end{aligned}
$$

Obviously, for $i=1, \ldots, N, j=1, \ldots, M$, for all $\tau^{I} \in$ $\Omega^{N}$ and all $\Psi=(\psi, \eta) \in \Lambda$, there holds

$$
\begin{aligned}
& \min _{v_{i j} \geq 0}\left(c_{i j}^{I I}\left(\tau_{i}^{I}, \tau_{j}^{I I}\right)-\psi_{i}-\eta_{j}\right) v_{i j} \\
& = \begin{cases}0, & c_{i j}^{I I}\left(\tau_{i}^{I}, \tau_{j}^{I I}\right)-\psi_{i}-\eta_{j} \geq 0, \\
-\infty, & c_{i j}^{I I}\left(\tau_{i}^{I}, \tau_{j}^{I I}\right)-\psi_{i}-\eta_{j}<0 .\end{cases}
\end{aligned}
$$

Since the dual problem (4) assumes maximizing the functional (8) on set $\Lambda$, it is reasonable to search the maximum on a set of those $\Psi=(\psi, \eta)$ from $\Lambda$, for which $c_{i j}^{I I}\left(\tau_{i}^{I}, \tau_{j}^{I I}\right)-\psi_{i}-\eta_{j} \geq 0$, only. Therefore, after the transformations performed, the dual problem (4) can be formulated as follows:

$$
\begin{aligned}
& G(\Psi)=G(\psi, \eta)=\min _{\tau^{I} \in \Omega^{N}} G_{2}\left(\tau^{I},\{\psi, \eta\}\right) \\
& =\min _{\tau^{I} \in \Omega^{N}}\left[\int_{\Omega} \min _{k=1, \ldots, N}\left(c_{k}^{I}\left(x, \tau_{k}^{I}\right)+\psi_{k}\right) \rho(x) d x+\sum_{j=1}^{M} \eta_{j} b_{j}^{I I}\right] \\
& \rightarrow \max _{\Psi}
\end{aligned}
$$

subject to constraints

$$
\eta_{j} \leq c_{i j}^{I I}\left(\tau_{i}^{I}, \tau_{j}^{I I}\right)-\psi_{i}, i=1, \ldots, N, j=1, \ldots, M,
$$

Rewrite conditions (10) in the form of

$$
\eta_{j}=\min _{1 \leq k \leq N}\left(c_{k j}^{I I}\left(\tau_{k}^{I}, \tau_{j}^{I I}\right)-\psi_{k}\right)
$$

Substituting expressions (11) into formula (9), and excluding variable $\eta$ from the functional $G(\Psi)$, we obtain the dual problem (4) in the form of

$$
\begin{gathered}
G(\Psi)=\min _{\tau^{I} \in \Omega^{N}} G_{2}\left(\tau^{I}, \psi\right) \\
=\min _{\tau^{I} \in \Omega^{N}}\left[\int_{\Omega} \min _{k=1, \ldots, N}\left(c_{k}^{I}\left(x, \tau_{k}^{I}\right)+\psi_{k}\right) \rho(x) d x\right. \\
\left.+\sum_{j=1}^{M} b_{j}^{I I} \min _{k=1, \ldots, N}\left(c_{k j}^{I I}\left(\tau_{k}^{I}, \tau_{j}^{I I}\right)-\psi_{k}\right)\right] \rightarrow \max _{\psi} \\
\psi=\left(\psi_{1}, \ldots, \psi_{N}\right) \in E_{N}
\end{gathered}
$$

Thus, switching from the original Problem C (via the Lagrangian (2)) to the dual problem in the form of (12)-(13), we obtain a formula for component $\lambda_{\star}(\cdot)$ of an optimal solution of Problem $C$ in the form of (7), where, as $\left(\tau^{I}, \psi\right)$, an optimal solution $\left(\tau_{\star}^{I}, \psi^{\star}\right)$ of the dual problem (12)-(13) is chosen.

Next, substituting the found expression for $\lambda_{\star}(\cdot)$ from formula (7) with $\left(\tau^{I}, \psi\right)=\left(\tau_{\star}^{I}, \psi^{\star}\right)$ into the objective functional (1) of the Problem C, we come to a Problem D of finding components $v=\left(v_{11}, \ldots, v_{i j}, \ldots, v_{N M}\right)$ of the optimal solution to the original Problem C.

\section{Problem D.}

$$
I\left(\lambda_{\star}(\cdot), v\right)=\text { const }+\sum_{i=1}^{N} \sum_{j=1}^{M} c_{i j}^{I I}\left(\tau_{\star i}^{I}, \tau_{j}^{I I}\right) v_{i j} \rightarrow \min _{v}
$$

subject to constraints

$$
\begin{aligned}
& \sum_{j}^{M} v_{i j}=\int_{\Omega} \rho(x) \lambda_{\star i}(x) d x, i=1, \ldots, N ; \\
& \sum_{i=1}^{N} v_{i j}=b_{j}^{I I}, j=1, \ldots, M ; \\
& v_{i j} \geq 0, i=1, \ldots, N, j=1, \ldots, M,
\end{aligned}
$$

where the value const $=\int_{\Omega} \min _{k=1, \ldots, N}\left(c_{k}^{I}\left(x, \tau_{\star}^{I}\right)+\psi_{k}^{\star}\right) \rho(x) d x$ does not affect a point where the minimum of function $I\left(\lambda_{\star}(\cdot), v\right)$ is attained. 
Clearly, Problem D is a classical finite-dimensional transportation problem for which a balance constraint

$$
\int_{\Omega} \rho(x) d x=\sum_{i=1}^{N} \int_{\Omega_{i}} \rho(x) d x=\sum_{j=1}^{M} b_{j}^{I I}
$$

holds. To solve problem $\mathrm{D}$, one can apply the well-known potential method [17].

Let us formulate a theorem that sums up our arguments.

Theorem 2.1. A component $\lambda_{\star}(\cdot)=\left(\lambda_{\star_{1}}(\cdot), \ldots, \lambda_{\star_{i}}(\cdot)\right.$, $\left.\ldots, \lambda_{\star_{N}}(\cdot)\right)$ of an optimal solution of Problem $C$ is determined as follows:

$$
\lambda_{\star_{i}}(x)= \begin{cases}1, & \text { if } \quad c^{I}\left(x, \tau_{\star i}^{I}\right)+\psi_{i}^{\star} \leq c_{k}^{I}\left(x, \tau_{\star k}^{I}\right)+\psi_{k}^{\star}, \\ & i \neq k, k=1, \ldots, N, \\ 0, & \text { otherwise, }\end{cases}
$$

for all $i=1, \ldots, N$, and almost all $x \in \Omega$, where $\left(\tau_{\star 1}^{I}, \ldots, \tau_{\star_{N}}^{I}\right),\left(\psi_{1}^{\star}, \ldots, \psi_{N}^{\star}\right)$ is an optimal solution to the dual problem (4) in the form of (12), (13). A component $v_{\star}=$ $\left(v_{\star_{11}}, \ldots, v_{\star_{i j}}, \ldots, v_{\star_{N M}}\right)$ is sought as an optimal solution to the following finite-dimensional transportation problem by the potential method:

$$
\sum_{i=1}^{N} \sum_{j=1}^{M} c_{i j}^{I I}\left(\tau_{i}^{I}, \tau_{j}^{I I}\right) v_{i j} \rightarrow \min _{v}
$$

subject to constraints

$$
\begin{gathered}
\sum_{j}^{M} v_{i j}=\int_{\Omega} \rho(x) \lambda_{\star_{i}}(x) d x, \\
\sum_{i=1}^{N} v_{i j}=b_{j}^{I I}, \\
v_{i j} \geq 0,
\end{gathered}
$$

and the balance constraint (14).

Next, we present an algorithm for solving Problem C, which is based on Theorem 3.1. Two parts of this algorithm are: a) due to non-differentiability of function $G_{2}\left(\tau^{I}, \psi\right)$ ), it is one of the versions of the Shor's $r$-algorithm - the generalized pseudo-gradients method $[5,6]$ used to numerically solve the dual problem (12), (13); b) and the potential method [17] used to solve problem (14) - (18) of finding a component $v \star$ of an optimal solution of Problem C.

\subsection{Problem Solving Algorithm}

Before formulating an algorithm for solving Problem C, based on the above theorem, we define the $i$-th component $(i=1, \ldots, N)$ of a vector of generalized pseudo-gradient $g_{G_{2}}\left(\tau^{I}, \psi\right)=\left(g_{G_{2}}^{\tau^{I}}\left(\tau^{I}, \psi\right),-g_{G_{2}}^{\psi}\left(\tau^{I}, \psi\right)\right)=$ $\left(g_{G_{2}}^{\tau_{1}^{I}}\left(\tau^{I}, \psi\right), \ldots, g_{G_{2}}^{\tau_{N}^{I}}\left(\tau^{I}, \psi\right),-g_{G_{2}}^{\psi_{1}}\left(\tau^{I}, \psi\right), \ldots,-g_{G_{2}}^{\psi_{N}}\left(\tau^{I}, \psi\right)\right)$ of function $G_{2}\left(\tau^{I}, \psi\right)$ of problem (12), (13) at a point $\left(\tau^{I}, \psi\right)=\left(\tau_{1}^{I}, \ldots, \tau_{N}^{I}, \psi_{1}, \ldots, \psi_{N}\right)$ as follows:

$$
g_{G_{2}}^{\psi_{i}}\left(\tau^{I}, \psi\right)=\int_{\Omega} \rho(x) \lambda_{i}(x) d x+\sum_{j=1}^{M}\left(b_{j}^{I I} q_{i j}\right),
$$

where

$q_{i j}= \begin{cases}-1, & c_{i j}^{I I}\left(\tau_{i}^{I}, \tau_{j}^{I I}\right)-\psi_{i} \\ & =\min _{k=1, \ldots, N}\left(c_{k j}\left(\tau_{k}^{I}, \tau_{j}^{I I}\right)-\psi_{k}\right), i=1, \ldots, N, \\ & j=1, \ldots, M, \\ 0, & \text { otherwise; }\end{cases}$

$$
g_{G_{2}}^{\tau_{i}^{I}}=\int_{\Omega} \rho(x) g_{c^{I}}^{\tau_{i}^{I}}(\tau, x) \lambda_{i}(x) d x+\sum_{j=1}^{M}\left(b_{j}^{I I} r_{i j}\right),
$$

$i=1, \ldots, N$,

$$
r_{i j}= \begin{cases}g_{c^{I I}}^{\tau_{I}^{I}}\left(\tau_{i}^{I}, \tau_{j}^{I I}\right), & c_{i j}^{I I}\left(\tau_{i}^{I}, \tau_{j}^{I I}\right)-\psi_{i} \\ & =\min _{k=1, \ldots, N}\left(c_{k j}^{I I}\left(\tau_{k}^{I}, \tau_{j}^{I I}\right)-\psi_{k}\right), \\ & j=1, \ldots, M, \\ 0, & \text { otherwise, }\end{cases}
$$

$g_{c^{I}}^{\tau_{i}^{I}}\left(\tau^{I}, x\right)$ is the $i$-th component $(i=1, \ldots, N)$ of $N$ dimensional vector of the gradient $g_{c^{I}}^{\tau^{I}}\left(\tau^{I}, x\right)$ of function $c_{i}^{I}\left(x, \tau_{i}^{I}\right)$ at a point $\tau^{I}=\left(\tau_{1}^{I}, \ldots, \tau_{i}^{I}, \ldots, \tau_{N}^{I}\right) ; g_{c^{I I}}^{\tau_{i}^{I}}\left(\tau_{i}^{I}, \tau_{j}^{I I}\right)$ is $i$-th component $(i=1, \ldots, N)$ of $N$-dimensional vector of generalized gradient $g_{c^{I I}}^{\tau^{I}}\left(\tau_{i}^{I}, \tau_{j}^{I I}\right)$ of function $c_{i j}^{I I}\left(\tau_{i}^{I}, \tau_{j}^{I I}\right)$ at a point $\tau^{I}=\left(\tau_{1}^{I}, \ldots, \tau_{i}^{I}, \ldots, \tau_{N}^{I}\right)$;

$$
\lambda_{i}(x)= \begin{cases}1, & \text { if } \quad c^{I}\left(x, \tau_{i}^{I}\right)+\psi_{i}^{\star} \leq c_{k}^{I}\left(x, \tau_{k}^{I}\right)+\psi_{k}, \\ & i \neq k, \text { a.e. for } x \in \Omega, \quad k=1, \ldots, N, \\ 0, & \text { otherwise. }\end{cases}
$$

Let us describe the algorithm.

\section{Algorithm}

\section{Preliminary stage}

Enclose the domain $\Omega$ in an $n$-dimensional parallelepiped with sides parallel to axes of the Cartesian coordinate system, and assume that $\rho(x)=0$ if $x \in \Pi \backslash \Omega$. Cover the 
parallelepiped with a rectangular grid and set an initial approximation $\tau^{I}=\tau^{I^{(0)}}, \psi=\psi^{(0)}$. Calculate a value of $\lambda^{(0)}(x)$ at the grid nodes by formulas (21) with $\tau^{I}=\tau^{I^{(0)}}$, $\psi=\psi^{(0)}$. Evaluate the generalized pseudo-gradient vector $g_{G_{2}}\left(\tau^{I}, \psi\right)$ at the grid nodes using formulas (19)-(20) with $\tau^{I}=\tau^{I^{(0)}}, \psi=\psi^{(0)}, \lambda(x)=\lambda^{(0)}(x)$. Choose an initial trial step $h_{0}>0$ ( $h_{0}$ is typically chosen equal to 1$)$.

The first step of the algorithm we carry out according to the formulas

$$
\begin{aligned}
& \tau^{I(1)}=\mathrm{P}_{\Omega^{N}}\left[\tau^{I(0)}-h_{0} g_{G_{2}}^{\tau^{I}}\left(\tau^{I(0)}, \psi^{(0)}\right)\right], \\
& \psi^{(1)}=\psi^{(0)}+h_{0} g_{G_{2}}^{\psi}\left(\tau^{I(0)}, \psi^{(0)}\right),
\end{aligned}
$$

where $\mathrm{P}_{\Omega^{N}}$ is a projection operator on $\Omega^{N}$.

Suppose that, after $k(k=1,2, \ldots)$ steps of the algorithm, values $\tau^{I(k)}, \psi^{(k)}, \lambda^{(k-1)}(x)$ at the grid nodes have already been obtained as a result of calculations.

Describe the $(k+1)$-th step of the algorithm.

1. Calculate values $\lambda^{(k)}(x)$ at the grid nodes using formulas (21) with , $\psi=\psi^{(k)}$.

2. Evaluate a vector $g_{G_{2}}\left(\tau^{I}, \psi\right)$ at the grid nodes using formulas (19)-(20) with $\tau^{I}=\tau^{I(k)}, \psi=\psi^{(k)}, \lambda(x)=$ $\lambda^{(k)}(x)$.

3. Carry out the $(k+1)$-th step of the Shor's $r$-algorithm [6], the iteration formula of which is

$$
\begin{aligned}
& \tau^{I(k+1)}=\mathrm{P}_{\Omega^{N}}\left[\tau^{I(k)}-h_{k} B_{k+1}^{\tau^{I}} \tilde{g}_{G_{2}}^{\tau^{I}}\right], \\
& \psi^{(k+1)}=\psi^{(k)}+h_{k} B_{k+1}^{\psi} \tilde{g}_{G_{2}}^{\psi},
\end{aligned}
$$

where $B_{k+1}^{\tau^{I}}, B_{k+1}^{\psi}$ are operators mapping the transformed space into the original space $E_{N}$, herewith $B_{0}^{\tau^{I}}=I_{N}, B_{0}^{\psi}=I_{N}$ (the identity matrix); $\tilde{\boldsymbol{g}}_{G_{2}}^{\tau^{I}}=$ $B_{k+1}^{\star \tau^{I}} \boldsymbol{g}_{G_{2}}^{\tau^{I}}\left(\tau^{I(k)}, \psi^{(k)}\right), \tilde{g}_{G_{2}}^{\psi}=B_{k+1}^{\star} \psi \tilde{g}_{G_{2}}^{\psi}\left(\tau^{I(k)}, \psi^{(k)}\right) ; h_{k}$ is a step chosen from the condition of the minimum of difference $\left[G_{2}\left(\tau^{I(k-1)}, \psi^{(k)}\right)-G_{2}\left(\tau^{I(k)}, \psi^{(k-1)}\right)\right]$ in the direction of generalized anti pseudo-gradient $-g_{G_{2}}\left(\tau^{I}, \psi\right)$ in the transformed space.

4. If the constraint

$$
\left\|\left(\tau^{I(k+1)}, \psi^{(k+1)}\right)-\left(\tau^{I(k)}, \psi^{(k)}\right)\right\| \leq \varepsilon
$$

does not hold, we proceed to the $(k+2)$-th step of the algorithm, otherwise to step 5.

5. Let us assume that $\tau_{\star}^{I}=\tau^{I(l)}, \psi^{\star}=\psi^{(l)}, \lambda_{\star}(x)=$ $\lambda^{(l)}(x)$, where $l$ is a number of iterations, where the termination criterion (22) holds.

6. Solving the transportation problem by the potential method with $\lambda(x)=\lambda_{\star}(x), \tau^{I}=\tau_{\star}^{I}$ and $\psi=\psi^{\star}$, we find $v_{\star}=\left(v_{*} 11, \ldots, v_{\star} N M\right)$.
7. Calculate the optimal value of the objective functional $G_{2}\left(\tau^{I}, \psi\right)$ of the dual problem (12), (13) with $\tau^{I}=\tau_{\star}^{I}, \psi=\psi^{\star}$ and, for verifying correctness, the optimal value of the objective functional (1) of Problem $\mathrm{C}$ using the formula

$$
\begin{aligned}
& I\left(\lambda_{\star}(\cdot), \tau_{\star}^{I}, v_{\star}\right) \\
& =\sum_{i=1}^{N} \int_{\Omega} c_{i}^{I}\left(x, \tau_{\star i}^{I}\right) \rho(x) \lambda_{\star i}(x) d x \\
& +\sum_{i=1}^{N} \sum_{j=1}^{M} c_{i j}^{I I}\left(\tau_{\star i}^{I}, \tau_{j}^{I I}\right) v_{\star i j}
\end{aligned}
$$

The algorithm has been described. It was implemented in a solution to the following two model problems.

\section{Results}

\subsection{Model problem 1}

Let a supplier of a homogeneous resource (a raw material), continuously allocated with a density $\rho(x)=1$ in a domain $\Omega=\left\{x=\left(x^{(1)}, x^{(2)}\right): 0 \leq x^{(1)} \leq 1,0 \leq x^{(2)} \leq 1\right\}$, delivers it to two points (of stage I) for primary processing (storage). At the same time, coordinates $\tau_{i}^{I}=\left(\tau_{i}^{I(1)}, \tau_{i}^{I(2)}\right)$, $i=1,2$, of the location of these points may be unknown in advance. Suppose that coordinates $\tau_{j}^{I I}=\left(\tau_{j}^{I I(1)}, \tau_{j}^{I I(2)}\right)$, $j=\overline{1,6}$ of points of consumption (points of stage II) of the resource, processed (stored) at the points of stage I and the demand $b_{j}^{I I}$ for products at the $j$-th $(j=\overline{1,6})$ consumption point, are given. The cost of transporting a unit of the resource from a supplier with coordinates $x=\left(x^{(1)}, x^{(2)}\right)$ to the point $\tau_{i}^{I}=\left(\tau_{i}^{I(1)}, \tau_{i}^{I(2)}\right)$ of stage I is given in the form $c_{i}^{I}\left(x, \tau_{i}^{I}\right)=\sqrt{\left(x^{(1)}-\tau_{i}^{I(1)}\right)^{2}+\left(x^{(2)}-\tau_{i}^{I(2)}\right)^{2}}, \quad i=1,2$.

The costs of transporting a unit of production from the $i$ th point $\tau_{i}^{I}=\left(\tau_{i}^{I(1)}, \tau_{i}^{I(2)}\right)$ of stage I to the $j$-th point $\tau_{j}^{I I}=$ $\left(\tau_{j}^{I I(1)}, \tau_{j}^{I I(2)}\right)$ of stage II are given in the form

$$
\begin{aligned}
& c_{i j}^{I I}\left(\tau_{i}^{I}, \tau_{j}^{I I}\right)=\sqrt{\left(\tau_{i}^{I(1)}-\tau_{j}^{I I(1)}\right)^{2}+\left(\tau_{i}^{I(2)}-\tau_{j}^{I I(2)}\right)^{2}}, \\
& i=1,2, j=\overline{1,6} .
\end{aligned}
$$

In addition, we will assume that the capacity of the $i$-th $(i=1,2)$ point of stage $\mathrm{I}$ is determined by the total reserve of resource in the subdomain $\Omega_{i}$ of service of this point and should not exceed specified volumes $b_{i}^{I}: \int \rho(x) d x \leq b_{i}^{I}$, 
$i=1,2$. The numerical values of the initial data for the problem are presented in Table 1.

A set $\Omega$ of resource suppliers can be partitioned into subregions $\Omega_{i}$, each of which is serviced only by one $i$-th $(i=1,2)$ point of stage I, so that $\bigcup_{i=1}^{2} \Omega_{i}=\Omega$, mes $\left(\Omega_{1} \cap \Omega_{2}\right)=0$.

It is required to partition a set $\Omega$ of resource suppliers into their service subregions $\Omega_{1}, \Omega_{2}$ by two points of stage I, find coordinates of the location of points of stage I $\tau_{i}^{I}=\left(\tau_{i}^{I(1)}, \tau_{i}^{I(2)}\right), i=1,2$ in region $\Omega$ (if they are not specified) and determine the transportation volumes $v_{i j} \geq 0$, $i=1,2, j=\overline{1,6}$, from points $\tau_{i}^{I}, i=1,2$, of stage I to consumption points (points of stage II) $\tau_{j}^{I I}, j=\overline{1,6}$ to minimize the total cost of transporting the resource from suppliers to the primary processing points (points of stage I) and of delivering the processed resource from points of stage I to the final consumption points (points of stage II):

$$
\begin{aligned}
& F\left(\left\{\Omega_{1}, \Omega_{2}\right\},\left\{\tau_{1}^{I}, \tau_{2}^{I}\right\},\left\{v_{11}, \ldots, v_{26}\right\}\right) \\
& =\sum_{i=1}^{2} \int_{\Omega_{i}} c_{i}^{I}\left(x, \tau_{i}^{I}\right) \rho(x) d x+\sum_{i=1}^{2} \sum_{j=1}^{6} c_{i j}^{I I}\left(\tau_{i}^{I}, \tau_{j}^{I I}\right) v_{i j} \\
& \rightarrow \min ,
\end{aligned}
$$

herewith:

- all the processed product from all points of stage I should be exported to the points of stage II:

$$
\sum_{j=1}^{6} v_{i j}=\int_{\Omega_{i}} \rho(x) d x,
$$

- all the demands of points of stage II should be satisfied:

$$
\sum_{i=1}^{2} v_{i j}=b_{j}^{I I}
$$

- the balance constraint should be met:

$$
\sum_{i=1}^{2} \int_{\Omega_{i}} \rho(x) d x=\sum_{j=1}^{6} b_{j}^{I I} .
$$

As noted in the problem statement, coordinates $\tau_{i}^{I}=$ $\left(\tau_{i}^{I(1)}, \tau_{i}^{I(2)}\right), i=1,2$, of the points' location on stage I can be either known or not.

First, we present a solution of problem (23) - (27) using the algorithm described above for the case of given fixed coordinates $\tau_{i}^{I}=\left(\tau_{i}^{I(1)}, \tau_{i}^{I(2)}\right), i=1,2$, of the points' location on stage I, as shown in Figure 1. For variables $\psi=\left(\psi_{1}, \psi_{2}\right)$ of the dual problem (12) - (13), the initial values $\psi_{i}^{(0)}=0, i=1,2$ were chosen. If $\left\|\psi^{(k+1)}-\psi^{(k)}\right\| \leq \varepsilon$, then the iteration terminates, where $k(k=0,1,2, \ldots)$ is an iteration number; $\varepsilon>0$ is a given accuracy; $\|\cdot\|$ is the Euclidean norm.

As a result of the algorithm implementation with accuracy $\varepsilon=0.0001$, an optimal partitioning of set $\Omega$ of suppliers of a homogeneous resource into subsets $\Omega_{1}, \Omega_{2}$ was obtained after 18 iterations. Boundaries of the corresponding subsets are shown in Figure 1 with solid lines. For the case when coordinates of points of stage I are given, as can be seen in Figure 1, the whole volume of the processed resource is transported from the points of stage I to the points of stage II, i.e., the balance constraint (27) holds. Transportation volumes are indicated in Figure 1 above the corresponding arrows directed from the points of stage I towards the points of stage II.

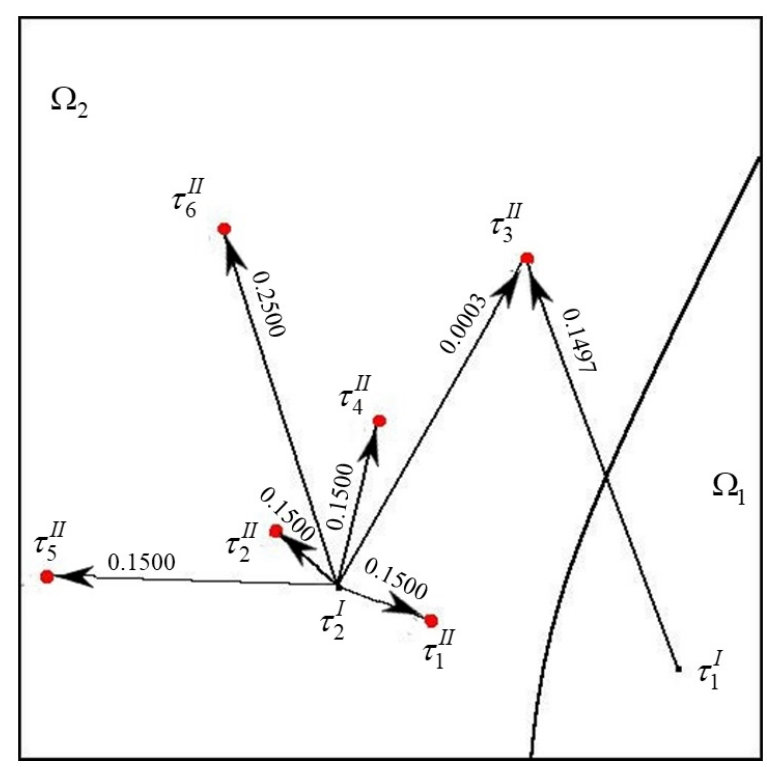

Figure 1: The model problem 1 optimal solution with given points of stage I

Numerical results of solving the model problem 1 for the case of given coordinates of stage I points are presented in the second column of Table 1. The optimal plan for transportation of the processed resource from $i$-th $(i=1,2)$ point of stage I to $j$-th $(j=\overline{1,6})$ point of stage II, is as follows:

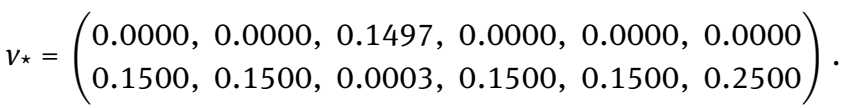


Table 1: Numerical results of solving the model problem 1

\begin{tabular}{|c|c|c|}
\hline & $\begin{array}{l}\text { Coordinates of points of } \\
\text { stage I are given }\end{array}$ & $\begin{array}{c}\text { Coordinates of points of } \\
\text { stage I are searched }\end{array}$ \\
\hline Given coordinates of points of stage I & $\begin{array}{l}\tau_{1}^{I}=(0.89 ; 0.12) \\
\tau_{2}^{I}=(0.43 ; 0.23)\end{array}$ & 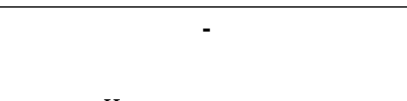 \\
\hline Given coordinates of points of stage II & \multirow{2}{*}{\multicolumn{2}{|c|}{$\begin{array}{c}\tau_{1}^{I I}=(0.55 ; 0.19) ; \tau_{2}^{I I}=(0.34 ; 0.31) ; \tau_{3}^{I I}=(0.68 ; 0.68) \\
\tau_{4}^{I I}=(0.48 ; 0.46) ; \tau_{5}^{I I}=(0.03 ; 0.25) ; \tau_{6}^{I I}=(0.27 ; 0.72) \\
b_{1}^{I I}=0.15 ; b_{2}^{I I}=0.15 ; b_{3}^{I I}=0.15 ; b_{4}^{I I}=0.15 ; b_{5}^{I I}=0.15 ; \\
b_{6}^{I I}=0.25\end{array}$}} \\
\hline Given demands in points of stage II & & \\
\hline Number of iterations & 18 & 41 \\
\hline Maximum value of the dual problem functional & $G\left(\psi^{\star}\right)=0.7611$ & $G\left(\psi^{\star}\right)=0.6507$ \\
\hline Minimum value of the primal problem functional & $I\left(\lambda_{\star}(\cdot), \tau_{\star}^{I}, v_{\star}\right)=0.7611$ & $I\left(\lambda_{\star}(\cdot), \tau_{\star}^{I}, v_{\star}\right)=0.6508$ \\
\hline Optimal capacities of each point of stage I & $b_{\star 1}^{I}=0.1497 ; b_{\star 2}^{I}=0.8503$ & $b_{\star 1}^{I}=0.6111 ; b_{\star 2}^{I}=0.3889$ \\
\hline Optimal coordinates of placed points of stage I & - & $\begin{array}{l}\tau_{\star 1}^{I}=(0.27 ; 0.72) \\
\tau_{\star 2}^{I}=(0.03 ; 0.25)\end{array}$ \\
\hline
\end{tabular}

In contrast to the previous case, where the points' coordinates were given, if the model problem 1 with a search of optimal coordinates of points $\tau_{i}^{I}, i=1,2$ of stage $\mathrm{I}$ is solved by the above algorithm, then, for variables $\tau_{i}^{I}$ and $\psi_{i}$ of the dual problem (12) - (13), the following initial values were chosen: $\tau_{i}^{I(0)}=0$ and $\psi_{i}^{(0)}=0, i=1$, 2. If $\left\|\left(\tau^{I(k+1)}, \psi^{(k+1)}\right)-\left(\tau^{I(k)}, \psi^{(k)}\right)\right\| \leq \varepsilon, k=0,1,2, \ldots$, then the iteration terminates.

As a result of the algorithm implementation with accuracy $\varepsilon=0.0001$, an optimal partitioning of set $\Omega$ of suppliers of a homogeneous resource into subsets $\Omega_{1}, \Omega_{2}$ was obtained after 41 iterations. Boundaries of the corresponding subsets are shown in Figure 2 with solid lines. As in the case of given coordinates of stage I points, the whole volume of the processed resource is transported from the stage I points to the stage II points, i.e., the balance constraint (27) holds.

The computational experiment results in solving the model problem 1 with a search of optimal coordinates of stage I points are given in the third column of Table 1 . The optimal plan for processed resource transportation from $i$ th $(i=1,2)$ point of stage I to $j$-th $(j=\overline{1,6})$ point of stage II, is as follows:

$v_{\star}=\left(\begin{array}{l}0.0612,0.0000,0.1500,0.1500,0.0000,0.2500 \\ 0.0888,0.1500,0.0000,0.0000,0.1500,0.0000\end{array}\right)$.

As a consequence of optimal placement of stage I points, the following results were obtained (see Figure 2 and Table 1): a) the optimal objective functional value for the model problem 1 with optimally placed points of stage I is less than the one, is points of stage I are given; b) the optimally placed points $\tau_{1}^{I}$ and $\tau_{2}^{I}$ of stage I coincided (within the given accuracy) with the points and $\tau_{5}^{I I}$ of stage

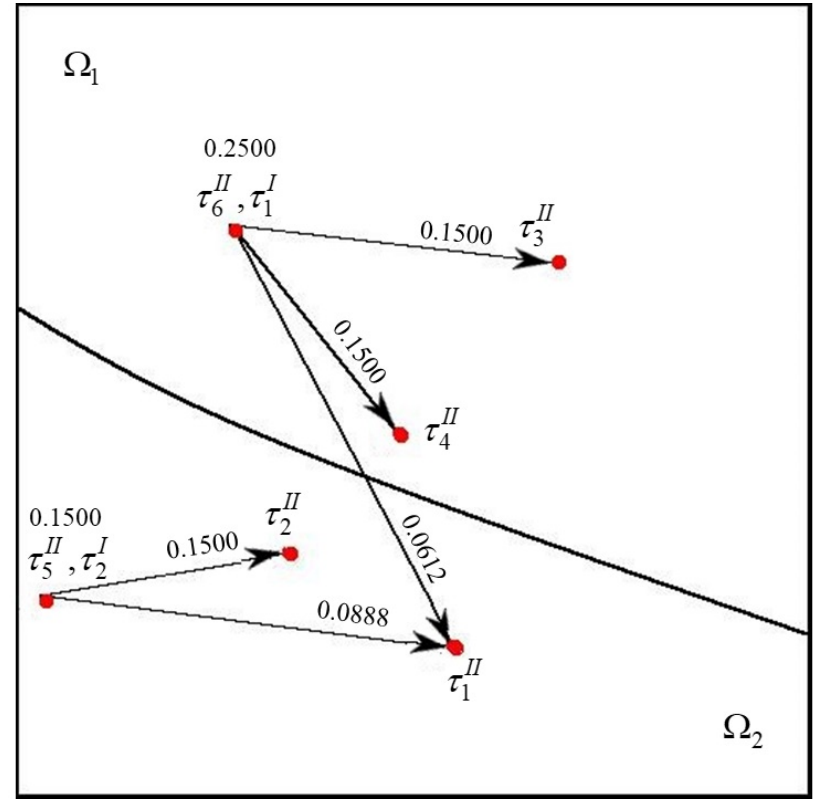

Figure 2: The model problem 1 optimal solution with optimally placed points of stage I

II, which is reasonable since the minimal transportation total cost is achieved if the points of stage I are placed directly at the points of stage II. At the same time, at those points of stage II, where the stage I points were placed, the demand constraint was satisfied, and a surplus of the processed resource at stage I points was distributed among the remaining points of stage II. This is shown in Figure 2 by corresponding arrows with the above-mentioned transportation volumes.

The reliability of calculations results of the described algorithm is confirmed by the coincidence (with a given 
accuracy) of the maximum value of the dual functional $G\left(\psi^{*}\right)$ with the minimum value of the primal functional $I\left(\lambda_{\star}(\cdot), \tau_{\star}^{I}, v_{\star}\right)$. It holds for the case of given points of stage I, as well as for optimally located points of stage I in the domain $\Omega$.

\subsection{Model problem 2}

This problem statement is similar to the model problem 1 statement. The only difference is that a homogeneous resource supplier delivers it to five points of stage I for primary processing (storage), and the processed resource is redistributed from the points of stage I to three points of stage II. For this problem, initial numerical values are presented in Table 2.

A solution of the model problem 2 by the above algorithm for the case of given coordinates of the location of stage I points is shown in Figure 3.

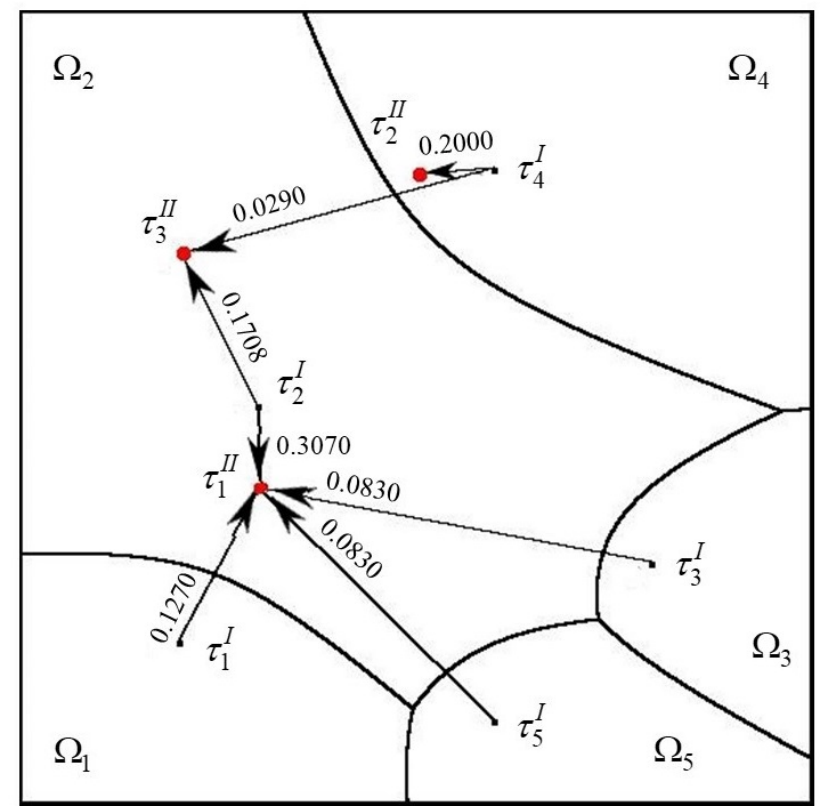

Figure 3: The model problem 2 optimal solutions with given points of stage I

As a result of the algorithm implementation with accuracy $\varepsilon=0.0001$, after 15 iterations, an optimal partitioning of set $\Omega$ of suppliers of a homogeneous resource into subsets $\Omega_{1}, \ldots, \Omega_{5}$ was obtained. Boundaries of the corresponding subsets are shown with solid lines in Figure 3.

Similar to the model problem 1, if coordinates of stage I points are given, the whole volume of the processed re- source is transported from the points of stage I to the points of stage II, i.e., the balance constraint holds.

Numerical results of solving the model problem 2 for the case of given coordinates of stage I points are presented in the second column of Table 2. The optimal plan for processed resource transportation from $i$-th $(i=\overline{1,5})$ point of stage I to $j$-th $(j=\overline{1,3})$ point of stage II, is as follows:

$$
v_{\star}=\left(\begin{array}{l}
0.1275,0.0000,0.0000 \\
0.3072,0.0000,0.1708 \\
0.0828,0.0000,0.0000 \\
0.0000,0.2000,0.0292 \\
0.0825,0.0000,0.0000
\end{array}\right) .
$$

If the model is solved with a search of optimal coordinates of stage I points by this algorithm, an optimal partition of set $\Omega$ of suppliers of a homogeneous resource into subsets $\Omega_{1}, \ldots, \Omega_{5}$ was obtained with accuracy $\varepsilon=$ 0.0001 after 237 iterations. The same as for given coordinates of stage I points, the whole volume of the resource is transported from points of stage I to points of stage II, respectively, the balance constraint holds.

The numerical results of solving the model problem 2 for the case of a search optimal coordinates of stage I points are presented in the third column of Table 2. The optimal plan for processed resource transportation from $i$ th $(i=\overline{1,5})$ point of stage I to $j$-th $(j=\overline{1,3})$ point of stage II, is as follows:

$$
v_{\star}=\left(\begin{array}{l}
0.5989,0.0000,0.0000 \\
0.0000,0.0005,0.0000 \\
0.0008,0.0003,0.0000 \\
0.0003,0.0000,0.2000 \\
0.0000,0.1992,0.0000
\end{array}\right) .
$$

Since during the allocation of processed resource from stage I points to stage II points, the resulting transportation volumes $v_{\star_{2 j}}$ and $v_{\star 3 j}(j=\overline{1,3})$ from points $\tau_{\star 2}^{I}$ and $\tau_{\star 3}^{I}$, respectively, are values comparable to the accuracy $\varepsilon=0.0001$ and due to the physical meaning of the problem, these values can be neglected with the indicated accuracy. Therefore, the solution of the model problem 2 with a search of optimal coordinates of stage I points location can have the form shown in Figure 5, where the 2nd and 3rd points of stage I do not function, because their volumes were neglected within the given accuracy.

Note that, for both model problems, the optimal placement of stage I points' results in (see Figures 4, 5 and Table 2): a) the objective functional optimal value of model problem 2 with optimally placed points of stage I is less than the optimal value of this functional for the case of given points of stage I; b) the optimally placed points $\tau_{1}^{I}$, $\tau_{4}^{I}$ and $\tau_{5}^{I}$ of stage I coincide (within the given accuracy) 
Table 2: Numerical results of solving the model problem 2

\begin{tabular}{|c|c|c|}
\hline & $\begin{array}{l}\text { Coordinates of points of } \\
\text { stage I are given }\end{array}$ & $\begin{array}{l}\text { Coordinates of points of } \\
\text { stage I are searched }\end{array}$ \\
\hline Coordinates of points of stage I & $\begin{array}{l}\tau_{1}^{I}=(0.20 ; 0.20) \\
\tau_{2}^{I}=(0.30 ; 0.50) \\
\tau_{3}^{I}=(0.80 ; 0.30) \\
\tau_{4}^{I}=(0.60 ; 0.80) \\
\tau_{5}^{I}=(0.60 ; 0.10)\end{array}$ & - \\
\hline Coordinates of points of stage II & \multirow{2}{*}{\multicolumn{2}{|c|}{$\begin{array}{c}\tau_{1}^{I I}=(0.30 ; 0.40) ; \tau_{2}^{I I}=(0.50 ; 0.80) ; \tau_{3}^{I I}=(0.20 ; 0.70) \\
b_{1}^{I I}=0.60 ; b_{2}^{I I}=0.20 ; b_{3}^{I I}=0.20\end{array}$}} \\
\hline Demands in points of stage II & & \\
\hline Number of iterations & 36 & 237 \\
\hline The maximum value of the dual problem functional & $G\left(\psi^{\star}\right)=0.4405$ & $G\left(\psi^{\star}\right)=0.3111$ \\
\hline The minimum value of the primal problem functional & $I\left(\lambda_{\star}(\cdot), \tau_{\star}^{I}, v_{\star}\right)=0.4405$ & $I\left(\lambda_{\star}(\cdot), \tau_{\star}^{I}, v_{\star}\right)=0.3113$ \\
\hline Optimal capacities of each point of stage I & $\begin{array}{c}b_{\star 1}^{I}=0.1275 ; b_{\star 2}^{I}=0.4780 ; \\
b_{\star 3}^{I}=0.0828 ; b_{\star 4}^{I}=0.2292 ; \\
b_{\star 5}^{I}=0.0825\end{array}$ & $\begin{array}{c}b_{\star 1}^{I}=0.5989 ; b_{\star 2}^{I}=0.0005 ; \\
b_{\star 3}^{I}=0.0011 ; b_{\star 4}^{I}=0.2003 ; \\
b_{\star 5}^{I}=0.1992\end{array}$ \\
\hline Optimal coordinates of placed points of stage I & 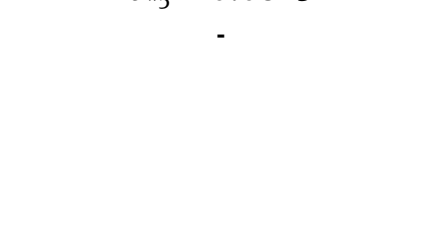 & $\begin{aligned} \tau_{\star 1}^{I} & =(0.3000 ; 0.4000) \\
\tau_{\star 2}^{I} & =(0.9594 ; 0.9964) ; \\
\tau_{\star 3}^{I} & =(0.3642 ; 0.9719) ; \\
\tau_{\star 4}^{I} & =(0.2000 ; 0.7000) ; \\
\tau_{\star 5}^{I} & =(0.5000 ; 0.8000)\end{aligned}$ \\
\hline
\end{tabular}

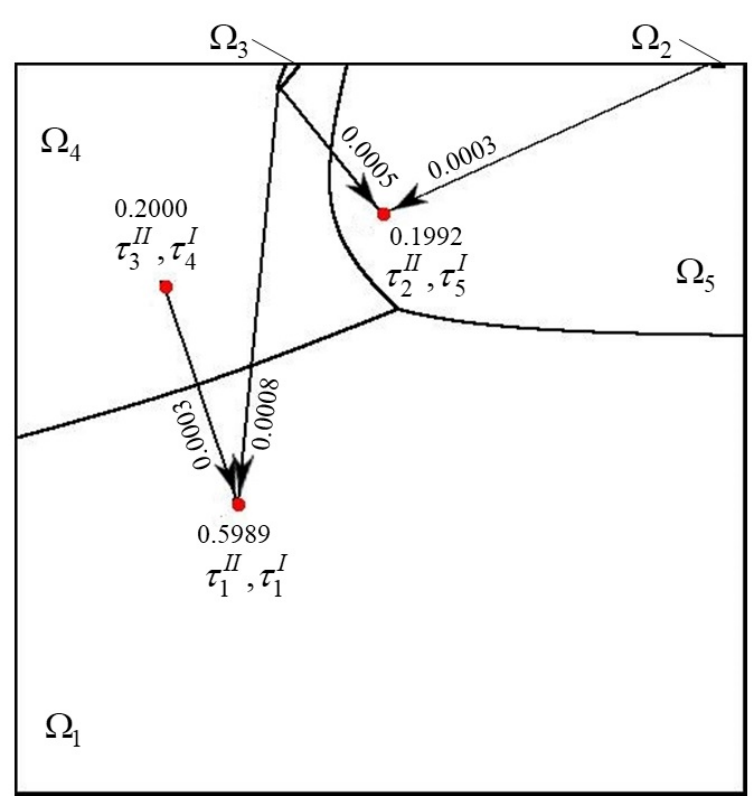

Figure 4: The model problem 2 optimal solution with optimally placed points of stage I

with the points $\tau_{1}^{I I}, \tau_{3}^{I I}$ and $\tau_{2}^{I I}$ of stage II, respectively. At the same time, the demand at those points of stage II, in which the stage I points were placed, was fully satisfied.

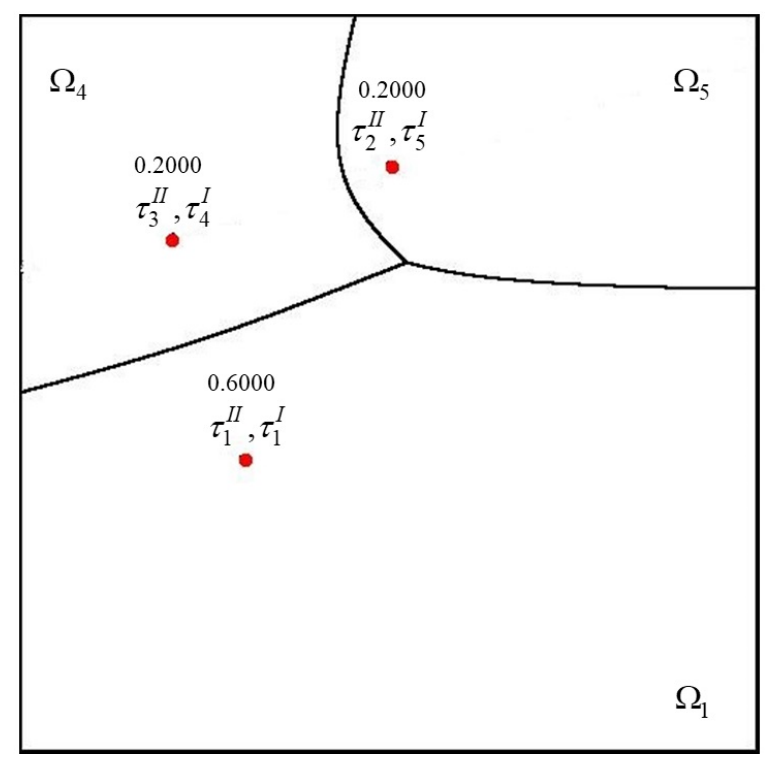

Figure 5: The model problem 2 optimal solution without points $\tau_{2}^{I}$ and $\tau_{3}^{I}$, whose volumes were neglected

\section{Discussion}

This paper is dedicated to the further development of the theory of optimal partitioning of sets in $n$-dimensional Euclidean space for the case of a two-stage continuousdiscrete linear optimal partitioning-allocation problem 
subject to equality constraints with the placement (finding coordinates) of subsets centers.

For a two-stage continuous-discrete optimal partitioning-allocation problem, a solution technique is based on an unified approach consisting of reducing an original infinite-dimensional problem of optimal partitioning-allocation to non-smooth (typically finitedimensional) optimization problems. For a numerical solution of these problems, effective methods of nondifferentiable optimization are applied, namely different versions of the Shor's $r$-algorithm.

For solving the problem under consideration, a method is proposed which utilizes optimal set-partitioning theory methods and the potential method for transportation problems. Based on the method, a numerical solution algorithm is developed. This algorithm was software implemented in the Intel Visual Fortran Compiler 18.0 for Windows, and its work is illustrated on two model problems.

For these models, computation results showed that a characteristic feature of the optimal solution of the twostage continuous-discrete partitioning-allocation problem considered in the paper is the coincidence (within the given accuracy) of optimally placed points of stage I with some points of stage II. Indeed, it is reasonable that the minimum total cost of products transportation is attained if the stage I points coincide with the stage II points.

\section{References}

[1] Corley H.W., Roberts S.D., A partitioning problem with applications in regional design, Operations Research, 20(5), 1972, 1010-1019.

[2] Corley H.W., Roberts S.D., Duality Relationships a Partitioning Problem, SIAM Journal of Applied Mathematics, 23(4), 1972, 490 494.

[3] Beyko I.V., Kiseleva E.M., The condition for the optimality of the boundary of the separation of zones of territorial service, The Application of Mathematical Methods to the Solution of Production and Economic Problems, Dnepropetrovsk, DGU, 1973, 53-55 (in Russian)

[4] Kiseleva E.M., A study of a class of optimal partitioning problems, PhD thesis, Dnepropetrovsk State University, Kiev, Ukraine, 1975 (in Russian)

[5] Shor N.Z., Minimization methods for non-differentiable functions, Springer series, Computational mathematics, Berlin, Springer-Verlag, vol. 3, 1985.

[6] Shor N.Z., Nondifferentiable optimization and polynomial problems, Boston, Dordrecht; London, Kluwer Academic Publishers, 1998.

[7] Stetsyuk P.I., Shor's r-algorithms: Theory and practice. In: Butenko S., Pardalos P.M., Shylo V. (Eds), Optimization Methods and Applications: In Honor of the $80^{\text {th }}$ Birthday of Ivan V. Sergienko, Springer, 2017, 495-520.
[8] Optimization methods and software, Special Issue "Nonsmooth optimization and related topics, dedicated to the memory of professor Shor N.”, 23 (1), 2008, 3-4, DOI: 10.1080/10556780701652368.

[9] Kiseleva E.M., The emergence and formation of the theory of optimal set partitioning for sets of the $n$ dimensional Euclidean space. Theory and application, Journal of Automation and Information Sciences, 50(9), 2018, 1-24, DOI: 10.1615/JAutomatInfScien.v50.i9.10.

[10] Kiseleva E.M., Stepanchuk T.F., On the efficiency of a global nondifferentiable optimization algorithm based on the method of optimal set partitioning, Journal of Global Optimization, 25, 2003, 209-235.

[11] Kiseleva E.M., Koriashkina L.S., Theory of continuous optimal set partitioning problems as a universal mathematical formalism for constructing voronoi diagrams and their generalizations. II. Algorithms for constructing Voronoi diagrams based on the theory of optimal set partitioning, Cybernetics and Systems Analysis, 51(4), 2015, 489-499, DOI: 10.1007/s10559-015-9740-y.

[12] Aurenhammer F., Klein R., Lee D.-T., Voronoi diagrams and delaunay triangulations, World Scientific Pub Co Inc, 2013.

[13] Kiseleva E.M., Lozovskaya L.I., Timoshenko E.V., Solution of continuous problems of optimal covering with spheres using optimal set-partition theory, Cybernetics and Systems Analysis, 45 (3), 2009, 421-437.

[14] Yakovlev S.V., The method of artificial dilation in problems of optimal packing of geometric objects, Cybernetics and Systems Analysis, 53 (5), 2017, 725-731.

[15] Yakovlev S.V., Formalizing spatial configuration optimization problems with the use of a special function class, Cybernetics and Systems Analysis, 55(4), 2019, 581-589.

[16] Murat A., Verter V., Laporte G., A continuous analysis framework for the solution of location-allocation problems with dense demand, Computers \& Operations Research, 37(1), 2010, 123-136.

[17] Gol'shtein E.G., Yudin D.B., Objectives of transport-type linear programming, Moscow, Nauka, 1969 (in Russian).

[18] Kiseleva E.M., Prytomanova O.M., Us S.A., Solving a two-stage continuous-discrete optimal partitioning-distribution problem with a given position of the subsets centers, Cybernetics and Systems Analysis, 56(1), 2020, 3-15.

[19] Stetsyuk P., Lyashko V., Mazyutynets G., Two-stage transportation problem and its AMPL-realization, NAUKMA Research Papers, Computer Science, 1, 2018, 14-20 (in Ukrainian).

[20] Drezner Z., Hamacher H.W., Facility location: application and theory, Berlin, Springer, 2001.

[21] Fengqi You, Grossmann I.E., Mixed-integer nonlinear programming models and algorithms for large-scale supply chain design with stochastic inventory management, Industrial and Engineering Chemistry Research, 47(2), 2008, 7802-7817.

[22] Gimadi E.Kh. Effective algorithms for solving the multi-stage placement problem on a chain, Discrete Analysis and Operations Research, 2 (4), 1995, 13-31 (in Russian)

[23] Kochetov Yu., Bilevel facility location: discrete models and computational methods, Proceedings of XXXVII Symposium on Operations Research, Tara, Serbia, 2010, 12-16. 